\title{
Contribution of Dataset Reduction Techniques to Tree-Simplification and Knowledge Discovery
}

\author{
Marc Sebban and Richard Nock \\ TRIVIA Research Team, French West Indies and Guiana University \\ Department of Mathematics and Computer Science, 95159 - Pointe-à-Pitre (France) \\ $\{$ msebban,rnock\}@univ-ag.fr
}

\begin{abstract}
In the Knowledge Discovery in Databases (KDD) field, the human comprehensibility of models is as important as the accuracy optimization. To address this problem, many methods have been proposed to simplify decision trees and improve their understandability. Among different classes of methods, we find strategies which deal with this problem by $a$ priori reducing the database, either through feature selection or case selection. At the same time, many other efficient selection algorithms have been developed in order to reduce storage requirements of casebased learning algorithms. Therefore, their original aim is not the tree simplification. Surprisingly, as far as we know, few works have attempted to exploit this wealth of efficient algorithms in favor of knowledge discovery. This is the aim of this paper. We analyze through large experiments and discussions the contribution of the state-of-the-art reduction techniques to tree simplification. Moreover, we propose an original mixed procedure which deals with the selection problem by jointly removing features and instances. We show that in some cases, this algorithm is very efficient to improve the standard post-pruning performances, used to combat the overfitting problem.
\end{abstract}

\section{Introduction}

While numerous predictive models have the only main objective to maximize the generalization accuracy (such as neural networks or nearest-neighbor algorithms), the aim of decision trees seems to be more complicated. In the field of Knowledge Discovery in Databases (KDD), even if the predictive accuracy is obviously important, the human comprehensibility of the model is actually also crucial. Unfortunately, with the huge development of the modern databases, this second goal is rarely reached, often resulting in deep incomprehensible trees. This large size is often explained by the two following reasons: (i) some target concepts can not be represented by trees, (ii) large modern databases can have a lot of noise, that causes overfitting. In such a context, researchers in statistics and computer science have proposed many methods for simplifying trees. Breslow and Aha [3] have proposed a clustering of methods in machine learning. They draw up the five following categories according to five simplification strategies:

D.A. Zighed, J. Komorowski, and J. Zytkow (Eds.): PKDD 2000, LNAI 1910, pp. 44-53, 2000.

(C) Springer-Verlag Berlin Heidelberg 2000 
control tree size, modify space of tests, modify search for tests, reduce dataset, transform into an alternative data structure.

In this paper, we focus only on dataset reduction techniques which belong to the fourth category. In this class of methods, two reduction strategies can be applied. The first one consists in removing irrelevant features before the tree induction process in order to build smaller trees. A study in [16] shows that such a strategy can be very efficient. Using C4.5 [12], the non deletion of weakly relevant features generates actually deeper decision trees with lower performances. Ad hoc algorithms has been proposed to deal with this phenomenon on decision trees $[1,5]$. These methods belong to a wider family of reduction algorithms, called wrapper models [9], which do not always use a decision tree representation.

The second strategy consists in deleting irrelevant instances before the construction of the tree. Such algorithms have been presented during the last decades in [11] (with a windowing strategy), [17] (with a sampling strategy) and [8] (with Robust $C 4.5$ decision trees). The Quinlan's strategy [11] consists in incrementally adding misclassified instances to an original learning sample and inducing a new decision tree until no cases are misclassified. To avoid to completely re-induce the tree, Utgoff [17] updates trees incrementally as each new case is added. John [8] chooses an opposite strategy by removing all instances misclassified by the current decision tree. Note that the tree is re-induced at each stage in the original algorithm, even if John claims that $R C 4.5$ could be modified with a scheme such as that proposed in [17]. This previous algorithm is one of the most efficient techniques, that is why we will take it as a reference in this paper (figure 1 recalls the pseudocode of $R C 4.5)$.

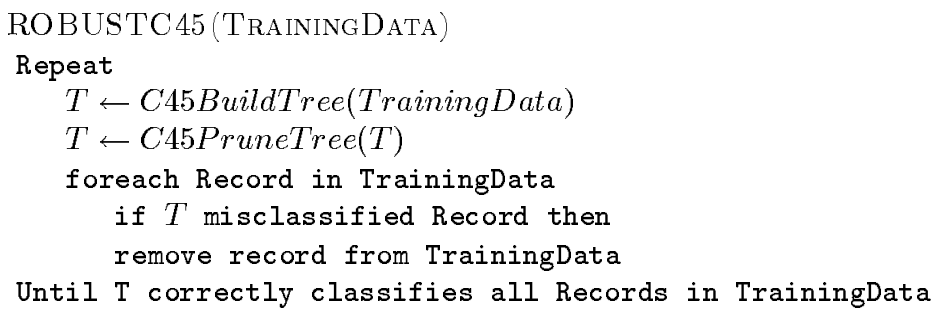

Fig. 1. Pseudocode of the ROBUST-C4.5 algorithm

At the same time, many other case-reduction techniques have been proposed, not for reducing tree size and improving human comprehensibility, but rather to limit the size required to store databases in memory $[4,6,7,15,18]$. These algorithms (often called Prototype Selection algorithms) have been developed in order to compensate for the drawback of case-based algorithms, such as the nearest-neighbor classifiers, well known to be very efficient, but also computationally expensive. As far as we know, no recent study has attempted to establish a close link between the best prototype selection procedures and tree simplifica- 
tion. No study has tried to compare algorithms for tree simplification, such as the John's Robust C4.5 Decision Trees with such algorithms. This behavior can be in fact doubly justified:

- The first reason (already mentioned above) comes from the main goal of the prototype selection algorithms: they are commonly used to reduce storage requirements imposed by expensive algorithms such as the $k$ Nearest-Neighbors $(k \mathrm{NN})$. Therefore, their reduction strategies are not a priori directed towards the construction of smaller trees.

- The second reason directly ensues from the first. Since most of these algorithms usually use a $k \mathrm{NN}$ classifier during the selection, their adaptation to tree simplification is a priori not natural.

Nevertheless, despite this established fact, three arguments prompt us to test them in order to simplify trees. First, while repeated tree inductions during the selection can be computationally costly on huge databases, some of prototype selection methods are known to be very fast. Even if incremental tree updates have been proposed [17], a local modification of a neighborhood graph is faster. Secondly, all the tree-simplification procedures optimize the accuracy during the selection by the induction algorithm. Recent theoretical [10] and empirical results [15] show that accuracy is not always the most suitable criterion to optimize. Since some of prototype selection algorithms take into account this phenomenon $[13,15]$, it would be interesting to analyze their effect for simplifying trees. Thirdly, the best prototype selection algorithms allow to dramatically reduce datasets, while not compromising the generalization accuracy. We think that some of them can be as efficient for simplifying trees.

In the first part of this paper, we will analyze the contribution of four stateof-the-art prototype selection algorithms: the Gates's Reduced Neighbor Rule [6], the consensus filters of Brodley and Friedl [4], the Wilson \& Martinez's RT3 [18] and the Sebban and Nock's PSRCG [15]. We think that they are representative of the evolution of the selection strategies in this field. After recalling the principles of these methods, we study their specificities in the field of tree simplification, and we statistically analyze their performances. Our goal is to answer to the two following questions:

- Which effect on tree simplification entails the nature of the optimized criterion during the selection, and the type of the learning algorithm used?

- What is the efficiency of these algorithms in comparison with procedures such as the Robust $C_{4.5}$ decision trees in terms of predictive accuracy, tree size, noise tolerance and learning speed?

Among the tested algorithms, we show that PSRCG [15], which does not optimize the accuracy, present performances statistically comparable to $R C 4.5$, with a possible adaptation to other tree induction algorithms, such as CART 
[2], or ID3 [11]. The information criterion optimized in $P S R C G$ is actually independent on a $a d$ hoc algorithm, and allows us to generalize its use for other decision trees. From this criterion, we propose in the second part a new mixed procedure which attempts to jointly delete irrelevant features and instances. A statistical study shows that our approach is suited to dramatically reduce tree sizes, while controlling the predictive accuracy, even in the presence of noise.

\section{Prototype Selection as a Tree Simplification Procedure}

\subsection{Prototype Selection Strategies}

In the wide family of prototype selection algorithms, we can distinguish three categories according to their selection strategy. For each category, we present the main methods (for more details see mentioned articles).

The first one only removes mislabeled instances or outliers. In such a category, the most efficient algorithm is probably the Consensus Filters [4]. This approach constructs a set of base-level detectors and uses them to identify mislabeled instances by consensus vote, i.e. all classifiers must agree to eliminate an instance. The base-level detectors can come from varied fields (neural network, induction tree, $k N N$, genetic algorithm, etc.).

The second category keeps only relevant instances, by removing not only outliers, but also useless instances, and examples at the class boundaries. Two examples of such algorithms are RT3 [18] and PSRCG [15]. They are known to belong to the most efficient prototype selection algorithms. In $R T 3$ an instance $\omega_{i}$ is removed if its removal does not hurt the classification of the instances remaining in the sample set, notably instances that have $\omega_{i}$ in their neighborhood (called associates). The algorithm is based on 2 principles: it uses a noise-filtering pass, which removes instances misclassified by their $k \mathrm{NN}$, that avoids "overfitting" the data; it removes instances in the center of clusters before border points, by sorting instances according to the distance from their nearest enemy (i.e. of a different class). In $P S R C G$, authors investigate the problem of selection as an information preserving problem. Rather than optimizing the accuracy of a classifier, they build a statistical information criterion based on a quadratic entropy computed from the nearest-neighbor topology. From neighbors linked by an edge to a given instance (including associates), PSRCG computes a quadratic entropy by taking into account the label of each neighbors. From this entropy (which conveys a local uncertainty), it deduces a global uncertainty of the learning sample. While an instance deletion is statistically significant, $P S R C G$ eliminates uninformative examples (see its statistical properties in [15]).

The last category presents an opposite strategy by keeping only misclassified instances. $C N N[7]$ and $R N N[6]$ were the pioneer works in this field. Their principle is based on the search for a consistent $(C N N)$ or a reduced $(R N N)$ subset, which correctly classifies all of the remaining instances in the sample set. 
Table 1. Predictive accuracy (Acc.), tree size (number of nodes \# N) and number of prototypes (\# pr.) on 21 benchmarks

\begin{tabular}{|c|c|c|c|c|c|c|c|c|c|c|c|c|c|c|c|}
\hline Dat & & 4.5 & & $\mathrm{RN}$ & $\mathrm{N}+\mathrm{C} 4$ & & Brod & $+c$ & $\mathrm{C} 4.5$ & $\mathrm{RT}$ & $3+\mathrm{C}$ & & $\mathrm{PSR}$ & $\mathrm{CG}+\mathrm{C}$ & 4.5 \\
\hline & $\overline{L S}$ & $\# \mathrm{~N}$ & Acc. & \# pr. & $\# \mathrm{~N}$ & Acc. & \# pr. & $\# \mathrm{~N}$ & Acc. & \# pr. & $\# \mathrm{~N}$ & Acc. & \# pr. & $\# \mathrm{~N}$ & Acc. \\
\hline & 0 & 31 & .1 & 209 & 29 & 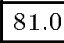 & 344 & 60 & 84.8 & 37 & 9 & 0 & 315 & 52 & 86.6 \\
\hline & & 106 & & & $\mathrm{U}$ & & & 75 & & 28 & 0 & & 00 & 91 & 12 \\
\hline & & 64 & & 50 & 14 & 1.0 & 3 & 32 & & 11 & 0 & & 79 & 5 & 92. \\
\hline & 5 & 34 & 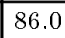 & 95 & 5 & 0 & 4 & 65 & 94.0 & 108 & 34 & .4 & 88 & 34 & 84.7 \\
\hline$\overline{e r}$ & 200 & 20 & 92.0 & 59 & 20 & 92.0 & 180 & 20 & 92.0 & 50 & 17 & 71. & 134 & 19 & 92.0 \\
\hline ch & 70 & 5 & 65.6 & 58 & 0 & & 52 & 2 & 63.0 & 6 & 2 & 63.0 & 40 & 21 & 73.0 \\
\hline & 500 & 5 & .6 & 1 & 27 & $\overline{9.0}$ & 411 & 27 & 9.0 & 29 & 2 & 64.5 & 357 & 91 & 69.0 \\
\hline la & 5 & 73 & 68.0 & 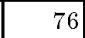 & 9 & 60.0 & 107 & 82 & 67.0 & 28 & 19 & : & 122 & 64 & 70.0 \\
\hline lep & 100 & 5 & 67.3 & 29 & 0 & & 89 & 5 & 67.3 & 3 & 0 & & 52 & l & 67.3 \\
\hline Hors & 200 & 49 & 82.1 & 92 & 36 & 5.0 & 160 & 49 & 81.5 & 17 & 2 & 83.3 & 186 & 45 & 81.5 \\
\hline onc & 185 & 124 & 83.6 & 40 & 27 & 66.0 & 163 & 25 & 91.4 & 19 & 2 & 87.9 & 140 & 46 & 88.8 \\
\hline 5 & 00 & 9 & 3.3 & 14 & 2 & 64.0 & 97 & 5 & 94.0 & 18 & 5 & 78. & 49 & 5 & 94.0 \\
\hline $\mathrm{ED}$ & 300 & 5 & 78.5 & 1 & 14 & 78.0 & 242 & 59 & 79.5 & 35 & 5 & 50.5 & 251 & 14 & 78.5 \\
\hline$\overline{\mathrm{EI}}$ & 300 & 20 & 89.0 & 114 & 12 & 75.0 & 266 & 38 & 85.0 & 24 & 14 & 75.0 & 193 & 9 & 87.5 \\
\hline Pima & 468 & 5 & 74.3 & 245 & 0 & & 369 & 27 & 75.0 & 25 & 5 & 73.7 & 438 & 5 & 74.7 \\
\hline Tic & 600 & 70 & $\begin{array}{l}75.7 \\
\end{array}$ & 218 & 2 & 60.0 & 506 & 76 & 79.1 & 164 & 17 & 62.0 & 533 & 98 & 81.6 \\
\hline & 400 & 9 & 69.7 & 8 & 2 & 61.0 & 335 & 65 & 74.0 & 52 & 23 & 70.6 & 375 & 20 & 70.6 \\
\hline Wa & 300 & 291 & 73.0 & 127 & 99 & 63.0 & 260 & 152 & 73.0 & 52 & 12 & 7.0 & 238 & 218 & 74.0 \\
\hline Wh & 235 & 9 & 90.5 & 25 & 5 & 87.0 & 226 & 2 & 94.0 & 15 & 2 & 94 & 42 & ? & 94.0 \\
\hline Wine & 100 & 12 & \begin{tabular}{|l|}
93.6 \\
\end{tabular} & 25 & 5 & 78.0 & 91 & 12 & 92.3 & 14 & 5 & 73.1 & 84 & 2 & 94.9 \\
\hline Kd6 & 400 & 96 & 83.5 & 157 & 0 & & 350 & 168 & 85.5 & 56 & 50 & 78.5 & 344 & 110 & 84.0 \\
\hline Avera & 00.4 & 49.6 & 80.7 & 119.1 & 14.7 & 73.8 & 259.9 & 49.8 & 81.0 & 37.7 & 10.7 & 72.5 & 206.9 & 41.0 & 81.5 \\
\hline
\end{tabular}

\subsection{Comparative Study}

The interest of this section consists in testing the listed methods and analyzing their effect on the tree size $a$ posteriori built with the $C 4.5$ algorithm. Deliberately, each algorithm has been applied using its original specificities (i.e. to reduce the storage requirement), without any methodological adaptation to tree simplification. Therefore, $R T 3$ and $R N N$ has been run using a $k \mathrm{NN}$ classifier (here $k=5$ ) during the selection (note that $C N N$ is not tested here because it contains the subset deduced by $R N N$ ). $P S R C G$ has been applied from a $5 N$ N graph. The problem for the Consensus Filters $(C F)$ was more complicated, because it requires to a priori provide the number of base-detectors and their nature. For this experimental study, we decided to take two base-detectors: a $5 \mathrm{NN}$ classifier and $C$ 4.5. These two classifiers must agree to remove an instance with $C F$.

We tested here a large panel of data bases, the large majority coming from the UCI Repository. The experimental set-up is the following: each data base is divided into a learning sample LS ( $2 / 3$ of instances), and a validation set VS (the remaining third). In our concern to control the time complexity, we did not used a cross-validation procedure. While it is not too expensive to compute distances 
for searching nearest-neighbors, the time requirement for building decision trees is actually higher. In our study, each prototype selection algorithm is applied on LS. C4.5 is then used to build the decision tree from the selected subset. VS is finally used to assess the predictive accuracy of the model. According to the tested association, several remarks can be made from table 1:

$R N N+C 4.5$ vs $C 4.5$ : The main specificity of the $R N N$ rule is the keeping of misclassified instances. This way to proceed results in a difficulty to build efficient trees because only overlapping and border points are used to identify relevant features. The results of $R N N+C 4.5$ confirm this drawback: while the average tree size is highly reduced (14.7 vs 49.6 nodes on average), that improves the model comprehensibility, the predictive accuracy is not controlled and decreases a lot (73.8 vs $80.7 \%)$. Therefore, we can not claim that $R N N+C 4.5$ is an efficient tree simplification procedure.

$R T 3+C 4.5$ vs $C 4.5$ : While $R T 3$ is particularly suited to reduce storage requirements of a $k \mathrm{NN}$ classifier without reducing the $k \mathrm{NN}$ predictive accuracy $[15,18$, it seems also to be inefficient for simplifying trees. $R T 3+C 4.5$ does not provide actually a good balance between the tree size reduction, i.e. the comprehensibility of the model ( 10.7 nodes on average vs 49.6 for $C 4.5$ ), and the control of the predictive accuracy which falls much (from $80.7 \%$ to 72.5 ). A Student paired t-test proves the significant superiority of $C 4.5$. This noting is in fact not amazing because $R T 3$ is originally optimized for the $k \mathrm{NN}$ classifier. The specificities of $R T 3$ based on distance calculations (noise-filtering pass removing instances misclassified by their $k \mathrm{NN}$; instance sorting according to the distance from their nearest enemy) make its adaptation for tree simplification difficult.

$C F+C 4.5$ vs $C 4.5: C F$ are known to be very efficient to improve $k$ NN performances because such classifiers are very sensitive to noise. On the other hand, its use for simplifying trees is a little of interest. Since $C F$ are only intended to remove mislabeled instances, the reduction of the data base size is limited (here, only $13.5 \%$ on average), resulting in near similar tree sizes on average (49.8 vs 49.6 ), with same predictive accuracies ( $81.0 \%$ vs 80.7 , that is not statistically significant with a critical risk near $40 \%$ ). One solution to improve $C F$ in such a context would consist in using only $C 4.5$ as base-level detector. But this way to proceed would amount in fact to applying the Robust-C 4.5 algorithm with only one pass.

$P S R C G+C 4.5$ vs $C 4.5$ : Incontestably, $P S R C G$ seems to be the most efficient algorithm for simplifying trees. Several criteria are actually improved in compar ison with the standard $C 4.5$ algorithm: (i) only $69 \%$ of the total number of instances are used for building the tree, that reduces the learning process; (ii) the number of nodes is $17.3 \%$ smaller than with the standard $C 4.5$, that allows a higher model comprehensibility; (iii) the predictive accuracy is improved (81.5\% vs 80.7$)$; using a Student paired t test over accuracies, we found that it is statistically significant with a critical risk near $12 \%$. 
Table 2. Accuracies for $C 4.5$ and $R C 4.5$ on 21 databases

\begin{tabular}{|c|c|c|c|}
\hline Accuracy C4.5 & Accuracy RC4.5 & Tree Size C4.5 & Tree Size RC4.5 \\
\hline 84.42 & 84.88 & 46.2 & 32.9 \\
\hline
\end{tabular}

$P S R C G+C 4.5$ vs Robust-C4.5: Since $P S R C G$ seems to be the most efficient procedure, we have to compare its performances to the Robust-C4.5 algorithm. We recall in table 2 the average results on accuracies and tree sizes presented in [8]. Note that even if benchmarks are not the same ( 8 among 21 are the same in the two experiments) and that John used a cross-validation procedure to assess the predictive accuracy, we can reasonably compare performances of the two methods. Even if the main advantage of $R C 4.5$ comes from a higher tree size reduction (28.8\% vs $17.3 \%$ with $P S R C G$ ), we can note that $P S R C G$ has four assets:

- using a Student paired t test over accuracies, and testing the superiority to $C 4.5$, we found that the critical risk with $R C 4.5$ is a little higher than with PSRCG $(14.5 \%$ vs $12 \%)$,

- While $R C 4.5$ requires the global update of the tree at each deletion stage, $P S R C G$ is based on fast local modifications of the $k \mathrm{NN}$ graph. The complexity of $P S R C G$ is then smaller than $R C 4.5$.

- our information criterion has statistical properties which establish a theoretical framework for halting selection,

- PSRCG does not depend on a given learning algorithm (as $C 4.5$ for $R C 4.5$ ). The $k \mathrm{NN}$ are actually used in $P S R C G$ only as an information graph, and not as a classifier.

\section{Joint Dataset Reduction for Tree Simplification}

We have shown in the previous section that thanks its theoretical properties, $P S R C G$ can constitute a good solution for simplifying trees. Until now, only instances have been at the center of our selection strategy to reduce tree size. However, we have proposed in [14] an original mixed procedure to take into account at the same time the two degrees of freedom of the selection problem: the number of features and the number of instances. This was the first attempt to take the reduction problem as a whole (features + instances) for a use in a $k \mathrm{NN}$ classification, and to establish a functional link between feature selection and instance selection. As far as we know, the endeavour to reduce tree size by removing features and instances in a single algorithm has not be achieved. We propose here to test our strategy for simplifying trees.

We recall in figure 2 the pseudocode of our algorithm, called $(F S+P S) R C G$. Note that $R C G$ assesses the gain of uncertainty due to the deletion of an instance 
Table 3. Contribution of our mixed procedure for simplifying different decision trees. The tree size is here presented by the number of induced rules \# $\mathrm{R}$

\begin{tabular}{|c|c|c|c|c|c|c|c|c|c|c|c|c|}
\hline Dataset & $\mathrm{C}$ & 4.5 & (FS+ & $+\mathrm{C} 4.5$ & $\mathrm{CA}$ & $\mathrm{RT}$ & $(\mathrm{FS}+1$ & CART & ID & 33 & $(\mathrm{FS}+$ & $8+$ ID3 \\
\hline & $\# \mathrm{R}$ & Acc. & $\# \mathrm{R}$ & Acc. & \# R & Acc. & $\# \mathrm{R}$ & Acc. & $\# \mathrm{R}$ & Acc. & $\# \mathrm{R}$ & Acc. \\
\hline Australian & 4 & 82.0 & 4 & 81.0 & 2 & 82.7 & 4 & 82.7 & 92 & 78.4 & 6 & 80.0 \\
\hline Bigpole & 3 & 69.5 & 3 & 69.5 & 11 & 63.5 & 6 & 64.0 & 60 & 56.0 & 59 & 61.8 \\
\hline Breast W. & 13 & 94.9 & 5 & 96.7 & 8 & 97.0 & 4 & 96.7 & 66 & 92.3 & 9 & 96.4 \\
\hline Brighton & 21 & 94.2 & 13 & 96.2 & 15 & 95.5 & 13 & 97.5 & 76 & 87.9 & 19 & 94.9 \\
\hline Car & 11 & 96.0 & 5 & 86.2 & 11 & 92.0 & 5 & 86.2 & 37 & 91.9 & 7 & 86.2 \\
\hline Dermato. & 8 & 92.0 & 6 & 92.0 & 6 & 91.0 & 6 & 89.0 & 34 & 84.4 & 11 & 90.0 \\
\hline Echocardio & 4 & 65.0 & 3 & 70.5 & 5 & 68.8 & 2 & 70.0 & 19 & 58.2 & 4 & 69.7 \\
\hline German & 3 & 69.6 & 4 & 70.8 & 7 & 67.0 & 7 & 68.8 & 145 & 64.0 & 7 & 68.8 \\
\hline Hepatit is & 8 & 70.0 & 2 & 70.0 & 2 & 70.0 & 2 & 70.0 & 15 & 70.0 & 3 & 64.5 \\
\hline Horse & 5 & 83.0 & 8 & 72.6 & 3 & 83.0 & 5 & 82.1 & 39 & 70.8 & 11 & 78.6 \\
\hline Iris & 3 & 96.0 & 3 & 84.0 & 3 & 96.0 & 3 & 84.0 & 16 & 91.0 & 3 & 84.0 \\
\hline LED +17 & 8 & 78.0 & 10 & 72.5 & 4 & 78.5 & 3 & 70.0 & 70 & 61.0 & 17 & 72.8 \\
\hline \begin{tabular}{|l} 
LED2 \\
\end{tabular} & 4 & 87.0 & 4 & 87.5 & 4 & 87.5 & 2 & 83.0 & 37 & 86.8 & 4 & 87.5 \\
\hline Pima & 3 & 74.6 & 7 & 70.5 & 8 & 72.0 & 14 & 65.7 & 98 & 66.8 & 18 & 62.0 \\
\hline Tic tac toe & 10 & 79.0 & 10 & 76.8 & 30 & 84.0 & 9 & 77.1 & 128 & 82.8 & 105 & 79.0 \\
\hline Vehicle & 7 & 73.0 & 7 & 71.7 & 5 & 72.8 & 6 & 72.6 & 88 & 68.6 & 14 & 73.1 \\
\hline Waves & 32 & 68.0 & 11 & 67.5 & 15 & 77.0 & 4 & 71.0 & 50 & 60.3 & 13 & 65.5 \\
\hline White H. & 3 & 94.0 & 2 & 94.0 & 2 & 94.0 & 2 & 94.0 & 41 & 89.8 & 2 & 94.0 \\
\hline Wine & 8 & 79.5 & 3 & 92.3 & 4 & 79.5 & 3 & 92.3 & 11 & 89.6 & 4 & 92.3 \\
\hline Average & 8.9 & 81.0 & 6.2 & 79.6 & 8.3 & 81.1 & 5.8 & 79.4 & 58.8 & 76.2 & \begin{tabular}{|l|}
17.1 \\
\end{tabular} & 78.6 \\
\hline
\end{tabular}

or a feature and $U_{l o c}(\omega)$ is the local uncertainty as presented in the previous section (for more details see [14]).

The $R C G$ specificities should allow us to use this procedure as a pre-process before the induction of decision trees by different algorithms, such as ID3 [11] or CART [2]. To test these other algorithms, we used the SIPINA software, developed in the computer science ERIC_Lyon laborator ${ }^{1}$, which collects the state-of-the-art tree induction algorithms. We tested our selection procedure on 20 datasets. Even if we kept the same experimental set-up, we decided here to assess the sensitivity of our algorithm to noise by adding artificially noise in data (by changing the output class of $10 \%$ of the learning instances). Results are presented in the table 3 , and discussed below:

$(F S+P S) R C G+C 4.5$ vs $C 4.5$ : the effect of our procedure as a pre-process to $C 4.5$ is interesting. Even if the predictive accuracy of $C_{4.5}$ is $1.4 \%$ reduced, this reduction is counterbalanced by an average reduction in the number of rules of about $30 \%$. In a field where the comprehensibility is crucial, such a size reduction keeping an excellent predictive accuracy would be very relevant. Using a Student paired test, we found that $C_{4.5}$ is significantly higher than $(F S+P S) R C G+C 4.5$ with a risk near $15 \%$.

\footnotetext{
${ }^{1}$ http://eric.univ-lyon2.fr
} 
$(F S+P S) R C G+C A R T$ vs $C A R T$ : the rule base with $C A R T$ is $30 \%$ smaller than with $(F S+P S) R C G+C A R T$. However, the predictive accuracy of the induced tree is statistically reduced with a smaller critical risk near $7.4 \%$.

$(F S+P S) R C G+I D 3$ vs ID3: the positive contribution of $(F S+P S) R C G$ is greatly presented by results on $I D 3$. Not only the number of rules is considerably reduced $(-71 \%)$, but also the generalization accuracy is significantly improved ( $78.6 \%$ vs $76.2 \%$ on average) that is highly significant with a risk near $3 \%$. These results are not amazing, because $I D 3$ does not use a post-pruning (as $C 4.5$ and $C A R T$ ), and is then very sensitive to noise because of the overfitting problem. By applying our mixed selection, it results in fact in deleting these noisy data and improving ID3 performances.

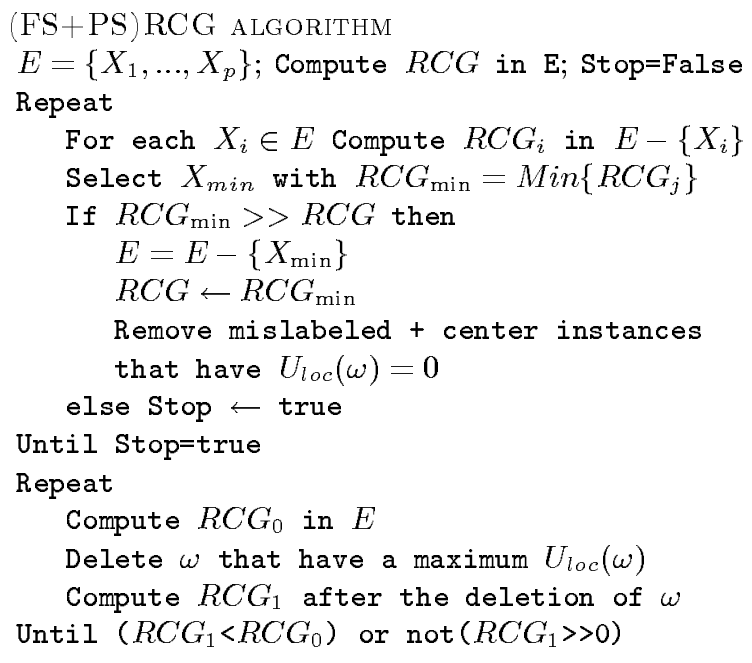

Fig. 2. Pseudocode of $(F S+P S) R C G$

\section{Conclusion}

With the development of modern data bases, dataset reduction becomes a major problem in many research fields such as in statistics or in computer science. Many reduction methods have been proposed for knowledge discovery in databases or for reducing computational costs of instance-based learning algorithms, without attempting to establish relations between these subfields. In this paper, we have tested some state-of-the-art prototype selection methods in order to simplify decision tree size. Experiments show that their contribution is not inconsiderable, and that future works will have to take into account these efficient algorithms for improving understandability of models. We also proposed an original procedure 
$(F S+P S) R C G$ to jointly reduce the number of features and instances. By this way to proceed, we emphasize the idea that future reduction algorithms will probably have to theoretically analyze the ways in which instance selection can aid the feature selection process. Moreover, we emphasize the need for studies designed to help understand and quantify the relationship that relates feature and prototype selections.

\section{References}

1. H. Almuallim and T.G. Dietterich. Learning with many irrelevant features. In Ninth National Conference on Artificial Intelligence, pages 547-552, 1991.

2. L. Breiman, J.H. Friedman, R.A. Olshen, and C.J Stone. Classification And Regression Trees. Chapman \& Hall, 1984.

3. L.A. Breslow and D.W. AHA. Simplifying decision trees: A survey. to appear in Knowledge Engineering Review, 1997.

4. C.E. BRODLEY and M.A. FrieDL. Identifying and eliminating mislabeled training instances. In Thirteen National Conference on Artificial Intelligence, 1996.

5. K.J. Cherkauer and J.W. Shavlik. Growing simpler decision trees to facilitate knowledge discovery. In Second International Conference on Knowledge Discovery and Data Mining, 1996.

6. G.W. Gates. The reduced nearest neighbor rule. IEEE Trans. Inform. Theory, pages 431-433, 1972.

7. P.E. HART. The condensed nearest neighbor rule. IEEE Trans. Inform. Theory, pages $515-516,1968$.

8. G.H. JoHN. Robust decision trees: Removing outliers from databases. In First International Conference on Knowledge Discovery and Data Mining, pages 174$179,1995$.

9. G.H. John, R. Kohavi, and K. Pfleger. Irrelevant features and the subset selection problem. In Eleventh International Conference on Machine Learning, pages $121-129,1994$.

10. M.J. KEARNS and Y. MANSOUR. On the boosting ability of top-down decision tree learning algorithms. Proceedings of the Twenty-Eighth Annual ACM Symposium on the Theory of Computing, pages 459-468, 1996.

11. J.R. QUINLAN. Induction of decision trees. Machine Learning, 1:81-106, 1986.

12. J.R. Quinlan. C4.5 : Programs for Machine Learning. Morgan Kaufmann, 1993.

13. R. E. SCHAPIRE and Y. SINGER. Improved boosting algorithms using confidencerated predictions. In Proceedings of the Eleventh Annual ACM Conference on Computational Learning Theory, pages 80-91, 1998.

14. M. SEBBAN and R. NoCK. Combining feature and example pruning by uncertainty minimization. In Sixteenth Conference on Uncertainty in Artificial Intelligence, 2000.

15. M. SEBBAN and R. NoCK. Instance pruning as an information preserving problem. In Seventeenth International Conference on Machine Learning, 2000.

16. THRUN ET AL. The monk's problem: a performance comparison of different learning algorithms. Technical report CMU-CS 91-197-Carnegie Mellon University, 1991.

17. P.E. UTGOFF. An improved algorithm for incremental induction of decision trees. In Eleventh Iternationla Conference on Machine Learning, pages 318-325, 1994.

18. D.R. WiLson and T.R. MARTINEZ. Instance pruning techniques. In Fourteenth International Conference on Machine Learning, pages 404-411, 1997. 